\title{
La radiografía de columna lumbar no modificó la evolución de los pacientes con lumbalgia
}

Radiography of the lumbar spine in primary care patients with low back pain: randomized controlled trial. Kendrick D, Fielding K, Bentley E y col. BMJ 2001; 322:400-5.

\section{Objetivo}

Evaluar la utilidad de la radiografía de columna lumbar en pacientes con lumbalgia, con respecto a la mejoría clínica y al grado de satisfacción con la atención médica recibida.

\section{Diseño}

Ensayo clínico aleatorizado (con técnica de sobres sellados), no ciego, con un seguimiento de nueve meses.

\section{Lugar}

Estudio multicéntrico realizado en 52 prácticas generales del Reino Unido.

\section{Pacientes}

Se incluyeron 421 pacientes con lumbalgia de una duración promedio de 10 semanas de evolución, (edad promedio: 40 años). Los criterios de exclusión fueron edad menor a 20 o mayor a 50 años, pérdida de peso o fiebre, terapia con corticoides, antecedentes de cáncer, tuberculosis, drogadicción EV, HIV positivos, embarazadas.

\section{Intervención}

El grupo intervención se realizó una radiografía de columna lumbar. Los pacientes de dicho grupo llevaban el resultado de la radiografía a su médico. Mientras, ambos grupos, tanto el grupo intervención como el grupo control, continuaban con el manejo habitual que su médico considerara conveniente (incluso el pedido de radiografía en el grupo control).

\section{Medición de resultados principales}

El evento primario a evaluar fue la diferencia promedio en la escala de Roland (adaptación de un cuestionario para evaluar impacto del dolor lumbar), y como eventos secundarios se incluyeron evaluación del dolor en una escala visual, satisfacción con la atención recibida, percepción general de salud, duración del dolor, uso de otros servicios de salud y cumplimiento de la prescripción médica. Enfermeras condujeron entrevistas personales antes de la aleatorización, a los tres y nueve meses para evaluar los distintos resultados.

\section{Conclusiones}

En general el grupo intervención no obtuvo mejores resultados que el grupo control en cuanto a mejoría clínica o utilización del sistema de salud. La única diferencia observada a los nueve meses fue la de estar algo más satisfecho con la atención recibida. No hubo diferencias entre los grupos en el puntaje de la escala de Roland independientemente de los hallazgos radiográficos. Es de destacar que el $80 \%$ de los pacientes, de haber tenido la posibilidad, hubieran elegido realizarse una radiografía.

Resultados Principales (Entre paréntesis rango intercuartilo a menos que este indicado porcentaje)

\begin{tabular}{|c|c|c|c|c|c|c|c|c|}
\hline & & & Iracteristicas & al inicio, a l & leses y a & & & \\
\hline Características Clínicas & & & & os tres mest & & & los nueve $m$ & \\
\hline & $\begin{array}{l}\text { Intervención } \\
(\mathrm{n}=210)\end{array}$ & $\begin{array}{l}\text { Control } \\
(\mathrm{n}=211)\end{array}$ & $\begin{array}{l}\text { Intervención } \\
(n=199)\end{array}$ & $\begin{array}{l}\text { Control } \\
(n=203)\end{array}$ & Valor p & $\begin{array}{l}\text { Intervención } \\
(\mathrm{n}=195)\end{array}$ & $\begin{array}{l}\text { Control } \\
(\mathrm{n}=199)\end{array}$ & Valor $p$ \\
\hline Continúa con dolor & & & $148(74 \%)$ & $132(65 \%)$ & 0,04 & $126(65 \%)$ & $113(57 \%)$ & 0,11 \\
\hline Escala de Roland & $7(4-11)$ & $8(4-12)$ & $4(1-8)$ & $3(1-7)$ & 0,05 & $3(0-7)$ & $2(0-6)$ & 0,06 \\
\hline Score de dolor & $2(1-2)$ & $2(1-2)$ & $1(1-2)$ & $1(0-2)$ & 0,06 & $1(0-2)$ & $1(0-2)$ & 0,17 \\
\hline Score percepción salud & $70(50-80)$ & $70(50-80)$ & $75(60-90)$ & $80(70-90)$ & 0,02 & $80(60-90)$ & $80(70-90)$ & 0,30 \\
\hline Satisfacción & $19(17-22)$ & $20(18-22)$ & $20(17-23)$ & $21(19-23)$ & 0,13 & $21(19-23)$ & $19(16-21)$ & $<0,01$ \\
\hline Se realizó Radiografía & & & $168(84 \%)$ & $15(7 \%)$ & & $171(88 \%)$ & $26(13 \%)$ & \\
\hline Visitó al médico & $2(1 \%)$ & 0 & $106(53 \%)$ & $60(30 \%)$ & $<0,01$ & $42(22 \%)$ & $47(24 \%)$ & 0.24 \\
\hline Cumplió Tratamiento & $135(64 \%)$ & $146(69 \%)$ & $63(32 \%)$ & $59(29 \%)$ & 0,57 & $56(29 \%)$ & $49(25 \%)$ & 0,36 \\
\hline Automedicación & $135(64 \%)$ & $154(73 \%)$ & $68(34 \%)$ & $67(33 \%)$ & 0,81 & $69(35 \%)$ & $57(29 \%)$ & 0,15 \\
\hline
\end{tabular}

Fuente de financiamiento: NHS Research and Development Health Technology Assesment Programme.

\section{Comentario}

Aproximadamente un $7 \%$ de la población adulta consulta por lumbalgia cada año. ${ }^{1}$ Las etiologías que una radiografía $(\mathrm{Rx})$ nos ayuda a descartar son menos del 10\%, 2 siendo incluso los hallazgos anormales frecuentes en personas asintomáticas. ${ }^{3}$ Por otro lado, también falla algunas veces en identificar causas importantes cuando estas están presentes. Existe evidencia que revela que el empleo de radiografías en los pacientes con lumbalgia simple sin signos de alarma (banderas rojas como fiebre, pérdida de peso, sospecha de cola de caballo, etc.) y hasta con seis semanas de dolor no alteran el curso de la enfermedad. En una encuesta realizada entre médicos de atención primaria, aproximadamente un $80 \%$ contestó que el motivo de solicitar una radiografía fue reasegurar al paciente o a ellos mismos. ${ }^{4}$ En el marco de este escenario la intención de este trabajo es evaluar la utilidad de la radiografía, no como test en función del diagnóstico, sino en relación a los resultados obtenidos en todo el proceso de atención que involucra también al tratamiento, al resultado obtenido, y a la satisfacción. Los resultados son claros: no se evidencia un beneficio en cuanto a la mejoría clínica pero si en cuanto a la satisfacción.

Sabiendo que la mayoría de las lumbalgias son autolimitadas y que el rédito de la $\mathrm{Rx}$, tanto diagnóstico como en relación a los resultados obtenidos es de escaso valor; será un desafío lograr el mismo grado de satisfacción percibido por los pacientes, prescindiendo de la radiografía. Recordemos que esto es aplicable cuando no haya signos de alarma que nos indiquen lo contrario (Banderas Rojas).

\section{Dr. Sebastián García Martí [ Construir Salud ]}

\section{Referencias}

1 Royal College of General Practitioners OoPCaS, and the Department of Health. Morbidity statistics from general practice. Fourth national study 1991-1992. London: HMSO, 1995.

2 Evaluating and Managing Acute Low Back Pain in the Primary Care Setting. Atlas S, Deyo R. J Gen Intern Med. 2001 Feb 16(2):120-31.

3 Magnetic Resonance Imaging of the Lumbar Spine in People without Back Pain. Jensen M, Brant-Zawadzki M, Obuchowski Ny col. NEJM. 1994 Jul 14: $331: 69-73$.

4 Survey of general practitioners opinions on the role of radiology in patients with low back pain. Owen J, Rutt G, Keir M y col. Br J Gen Pract 1990; 40: 98-101. 